\title{
Guidance of Neuronal Growth Cones in the Grasshopper Embryo. III. Recognition of Specific Glial Pathways
}

\author{
Michael J. Bastiani and Corey S. Goodman \\ Department of Biological Sciences, Stanford University, Stanford, California 94305
}

In the previous 2 papers, we focused on the selective affinities that growth cones display for specific axonal pathways. Little is known, however, about how this orthogonal scaffold of axonal pathways in the CNS is established in the first place, and what, if any, role glia might play in these events.

Here we show an important relationship between pioneering growth cones and primitive glial cells in the developing longitudinal connectives and peripheral nerve roots of the grasshopper embryo. We describe a preformed glial pathway for the formation of the intersegmental nerve, one of the major roots exiting the CNS. The growth cones that pioneer this nerve display a selective affinity for the segment boundary cell (SBC), a primitive glial cell that establishes the location of this nerve root. Similar glial cells are also found along the pathway where the longitudinal connectives form, and they too may play an important role in the formation of the first longitudinal axonal pathways. Experimental analysis shows that when the $\mathrm{SBC}$ is ablated, the growth cones that normally turn laterally to pioneer the intersegmental nerve do not do so, thus confirming the importance of the guiding role of this glial cell.

We postulate that a simple orthogonal scaffold of primitive glia is involved in the initial patterning of axonal pathways within and exiting the insect CNS; this concept is remarkably similar to the blueprint hypothesis proposed by Singer et al. (1979) to explain the development of axon pathways in vertebrates.

Many of our previous studies on the guidance of neuronal growth cones in the CNS of the grasshopper embryo focused on the selective affinities that growth cones display for specific axonal pathways; these specificities give rise to stereotyped patterns of selective fasciculation and suggest that axonal pathways are differentially labeled by surface recognition molecules (Bastiani et al., 1984, 1986; du Lac et al., 1986; Raper et al., 1983a-c, 1984).

These studies, however, do not address the question of how this orthogonal scaffold of axonal pathways in the CNS is established in the first place. Little is known about the guidance cues involved in these early events. Bate and Grunewald (1981) were the first to examine the pioneering of the first longitudinal axon pathways in the grasshopper embryo and suggested that the MP1, dMP2, and vMP2 growth cones might be guided by some extrinsic axial cue associated with the basement membrane, upon which the first growth cones extend.

Subsequent studies on these same neurons and pathways suggested this and one other possible source for guidance cues (Bastiani and Goodman, 1984a, b; Bastiani et al., 1985b; Good-

\footnotetext{
Received Feb. 18, 1986; revised Apr. 25, 1986; accepted May 5, 1986.

We thank Frances Thomas for technical assistance. This research was supported by a Scholars Award from the F.E.S.N. Foundation (M.J.B.) and grants and awards from the NIH, NSF, March of Dimes, and the McKnight Foundation (C.S.G.).

Correspondence should be addressed to Corey $\mathrm{S}$. Goodman at the above address.

Copyright (C) 1986 Society for Neuroscience $0270-6474 / 86 / 123542-10 \$ 02.00 / 0$
}

man et al., 1982; Taghert et al., 1982): (1) a continuous pathway of positional or polarity cues along the basement membrane, and (2) a more punctate pathway in the form of specifically labeled neurons just under the basement membrane (landmark cells; in the PNS, also called guidepost cells or stepping stones).

Similar hypotheses have been suggested for the guidance of the pioneering growth cones in the PNS during insect development (e.g., Bate, 1976; Bentley and Caudy, 1983; Bentley and Keshishian, 1982; Berlot and Goodman, 1984; Blair and Palka, 1985a, b; Ho and Goodman, 1982; Jan et al., 1985; Nardi, 1983). Although these studies implicated non-neuronal cells and surfaces in the guidance of the pioneering growth cones, they provided little information about the possible role that glia might play in the formation of these early axonal pathways. In contrast, much is known about the role of primitive glial cells in the formation of the early axonal pathways in vertebrates.

In vertebrates, pioneering growth cones have a special relationship with primitive glial cells in the developing spinal cord (e.g., Kuwada, 1986; Singer et al., 1979), the developing ventral roots (Nordlander et al., 1981), the developing optic nerve (e.g., Silver and Sapiro, 1981; Silver and Sidman, 1980), the developing auditory system (Carney and Silver, 1983), and the developing corpus callosum (Silver et al., 1982). In the absence of the preformed glial pathway, the corpus callosum does not form (Silver et al., 1982); when the glia are replaced, the commissure forms (Silver and Ogawa, 1983).

On the basis of these types of observations, Singer et al. (1979) proposed the blueprint hypothesis of neuronal pathway patterning, which identifies the surfaces of the neuroepithelium and its derivative primitive glia as providing a series of longitudinally oriented pathways for early axonal outgrowth.

The present study was motivated by 2 questions. First, how do the pioneering growth cones establish the diverse and often orthogonal pattern of axonal pathways within and exiting the CNS of the grasshopper embryo? Second, what role, if any, do glial cells play in the formation of these early axonal pathways? Because so much was already known about the development of the first 3 longitudinal axon fascicles, the intersegmental nerve, and the 7 identified neurons whose axons they initially contain (see, for example, the first two papers in this series), we decided to focus our attention on the formation of these first pathways and the possible role played by primitive glial cells.

In this paper we show the role of a preformed glial pathway in the formation of the intersegmental nerve, one of the major roots for both motoneurons and sensory neurons exiting and entering the CNS. The growth cones that pioneer the intersegmental nerve display a selective affinity for the segment boundary cell (SBC) (Bastiani et al., 1985a; Goodman et al., 1984), a primitive glial cell that establishes the location of this nerve root. Similar glial cells are also found along the pathway where the longitudinal connectives form, and they too appear to play an important role in the formation of the first longitudinal axonal pathways. 


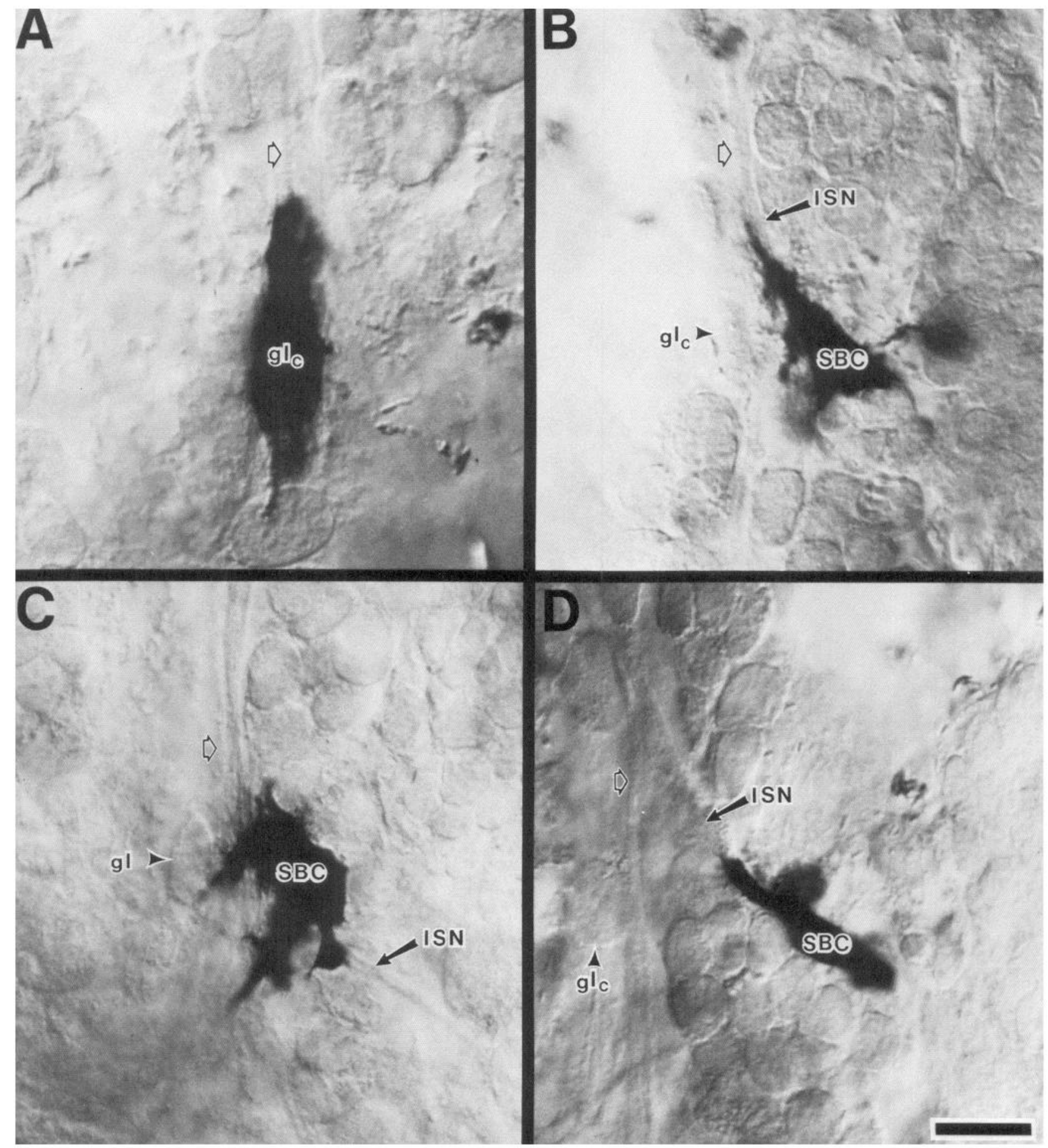

Figure 1. Morphology of primitive glial cells in the CNS of the grasshopper embryo, as revealed in these whole-mount preparations after injection of LY and HRP immunocytochemistry. Open arrows point to the location of the longitudinal axon fascicles of the connective and filled arrows point to the location of the intersegmental nerve $(I S N)$. $A$, Connective glial cell $\left(g l_{c}\right)$ at $34 \%$ of development. $B-D$, The segment boundary cell $(S B C)$ at 34,38 , and $45 \%$ of development, respectively, showing its changing morphology. Early in development, the $S B C$ is a flattened cell just under the dorsal basement membrane, with numerous processes and filopodia extending both laterally from its distal edge and longitudinally from its proximal side. The $I S N$ forms directly along the surface of the SBC. As the ISN forms, the SBC changes its shape as it withdraws many of its processes and becomes more tightly aligned along the nerve (compare $B$ with $D$ ). Scale bar, $20 \mu \mathrm{m}$. 


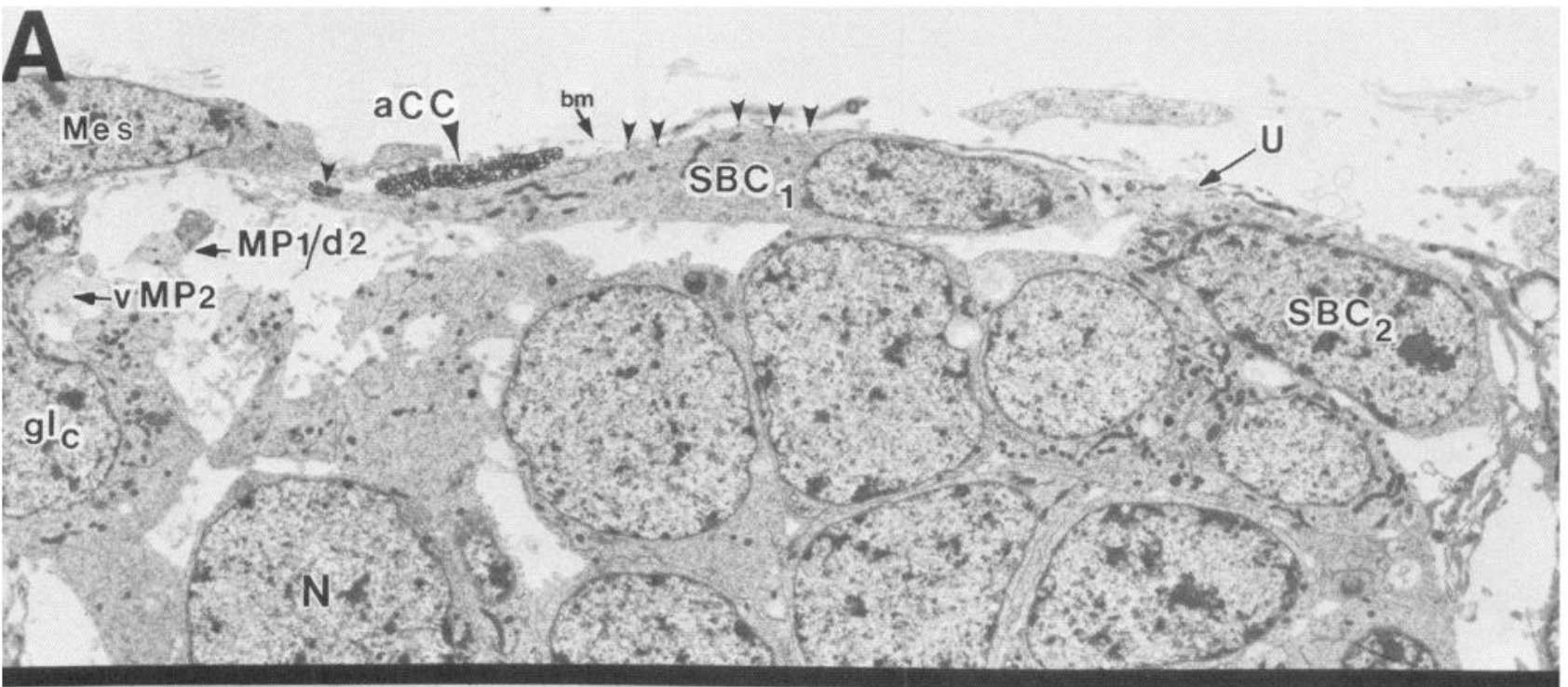

B
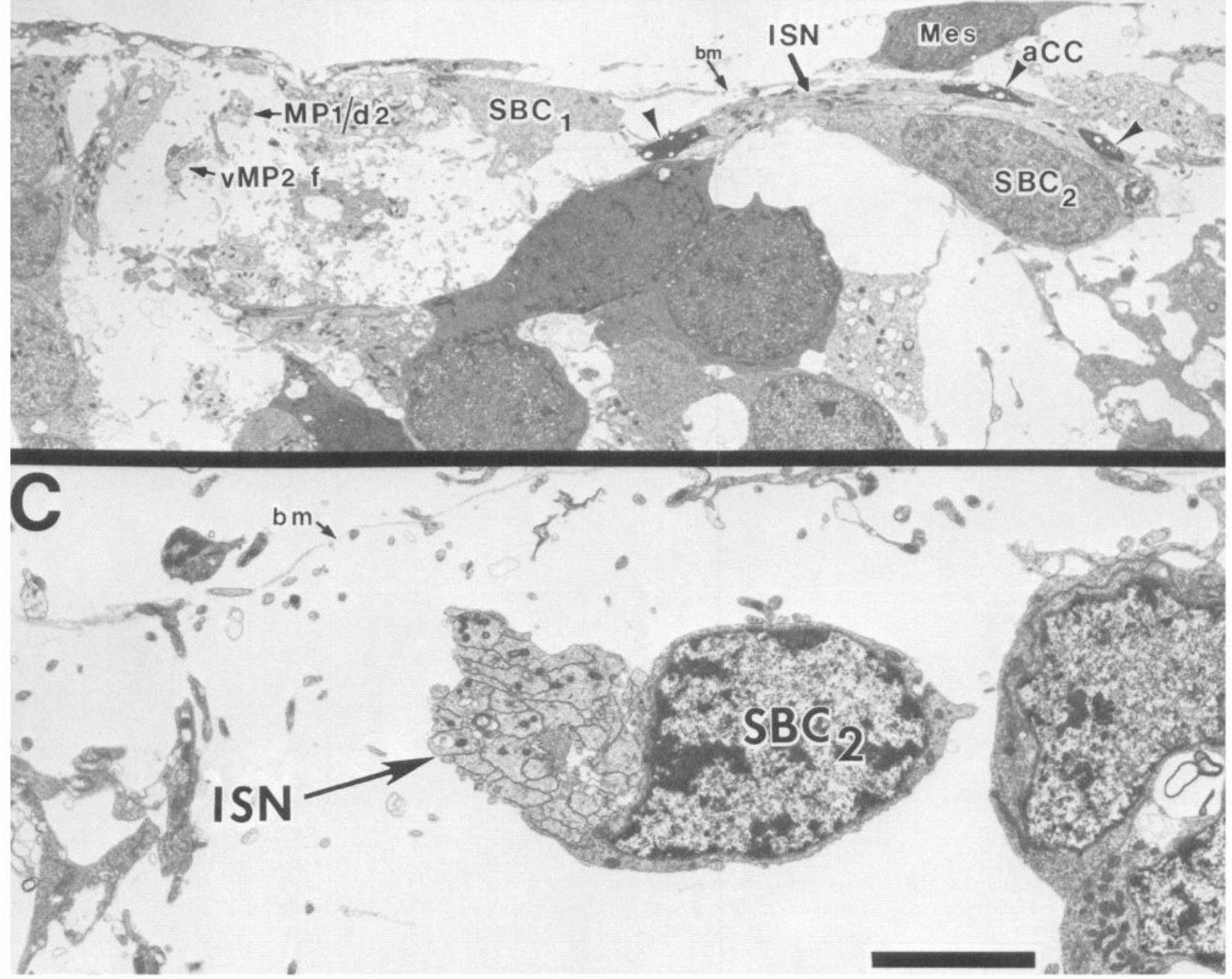

Figure 2. The SBC is a primitive glial cell which forms the proximal part of a preformed glial pathway for the IS nerve, one of the major peripheral nerve roots leaving the CNS of the grasshopper embryo. $A$, When the $U$ growth cones reach the segment border, they turn laterally along the SBC to pioneer the IS nerve (34\%). When the $a C C$ growth cone reaches the segment border, it too turns laterally (35\%), as shown here in a preparation in which the aCC has been filled with HRP. However, as shown here, although it extends posteriorly by fasciculating with the $U$ axons, it sometimes leaves their surface at the segment border and displays a higher affinity for the SBC. Most growth cones, however, appear uninterested in the SBC; 


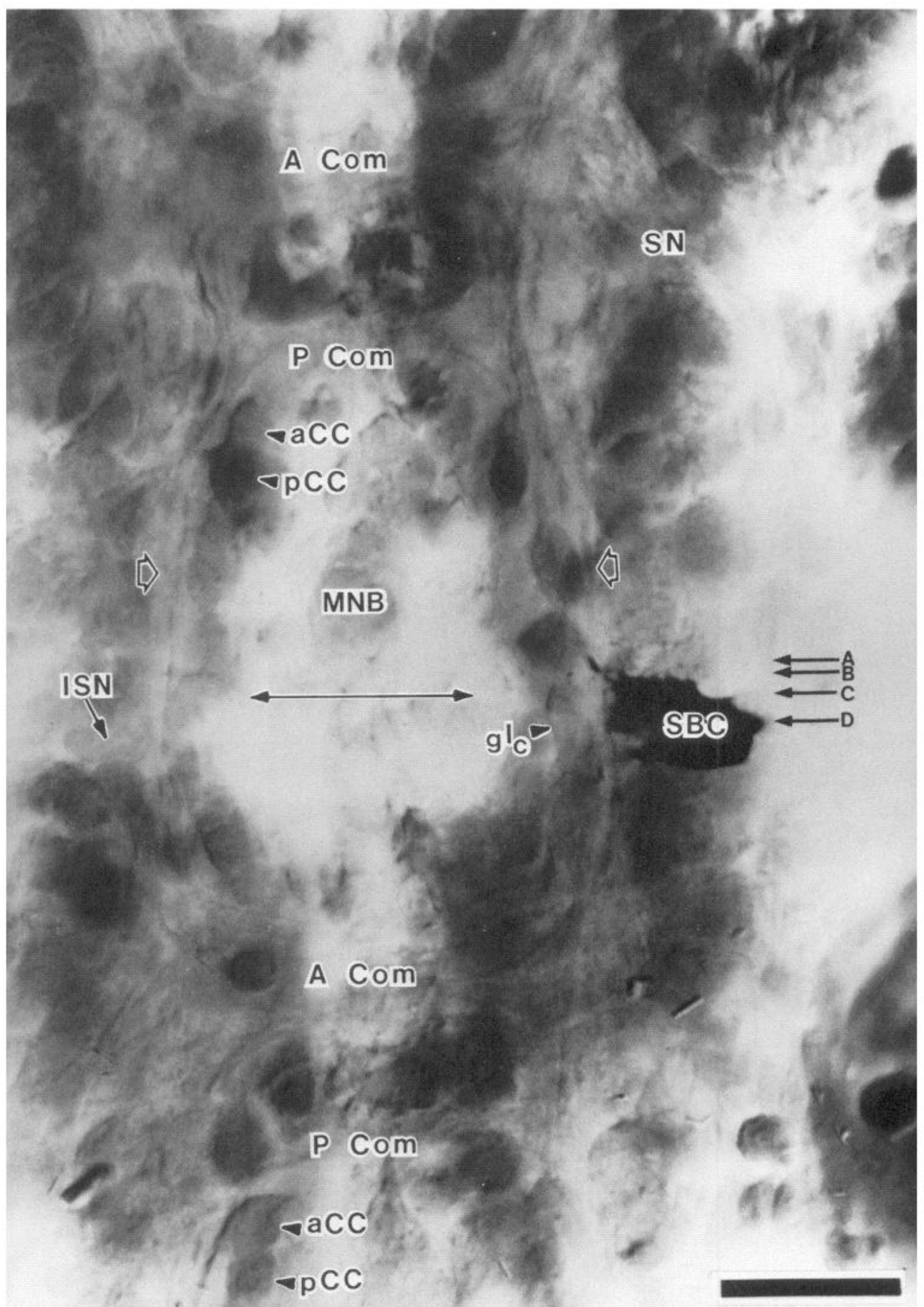

Figure 3. Location and morphology of $S B C$ in a whole-mount embryo (34\%) in which the SBC had been filled with HRP, photographed at the lightmicroscopic level, as shown here, and then processed for electron microscopy. Arrows at A, B, C, and D indicate the levels of the electron micrographs of this preparation shown in Figure 4. $g l_{c}$, Connective glia; $I S N$, IS nerve; $S N$, segmental nerve; $A C O M$, anterior commissure; $P$ Com, posterior commissure; $M N B$, median neuroblast; open arrows, location of longitudinal axon fascicles; horizontal line with arrows, segment border. Scale bar, $50 \mu \mathrm{m}$.
Experimental analysis shows that when the SBC is ablated, the growth cones that normally turn laterally to pioneer the intersegmental nerve do not do so, thus confirming the important guidance role played by this glial cell. We postulate that a simple orthogonal scaffold of primitive glia is involved in the initial patterning of axonal pathways within and exiting the insect CNS.

\section{Materials and Methods}

All methods used here are described in the first paper in this series.

for example, the $M P 1, d M P 2$, and $v M P 2$ growth cones extend longitudinally at the segment border through the same channel as the U1, U2, and aCC growth cones, without displaying any selective affinity for the SBC. Mes, Mesodermal cell; $g l_{c}$, connective glia; $b m$, basement membane; $N$, neuronal cell body. $B$, As the U1, U2, and $a C C$ growth cones continue to extend distally and exit the CNS, they leave the surface of the SBC and extend next along the surface of another identified cell, called $S B C_{2}$, which is also aligned along the presumptive IS nerve (ISN) pathway just distal to the $S B C_{1}$. In this $38 \%$ embryo, the aCC neuron has been filled with HRP. $v M P 2 f$, vMP2 fascicle, $C$, The SBC and its distal sibling $S B C_{2}$ are primitive glial cells; later in development, they differentiate into mature glial cells and enwrap the axons of the IS nerve (ISN) (45\%). Scale bar, $10 \mu \mathrm{m}(A, B) ; 5 \mu \mathrm{m}(C)$. 

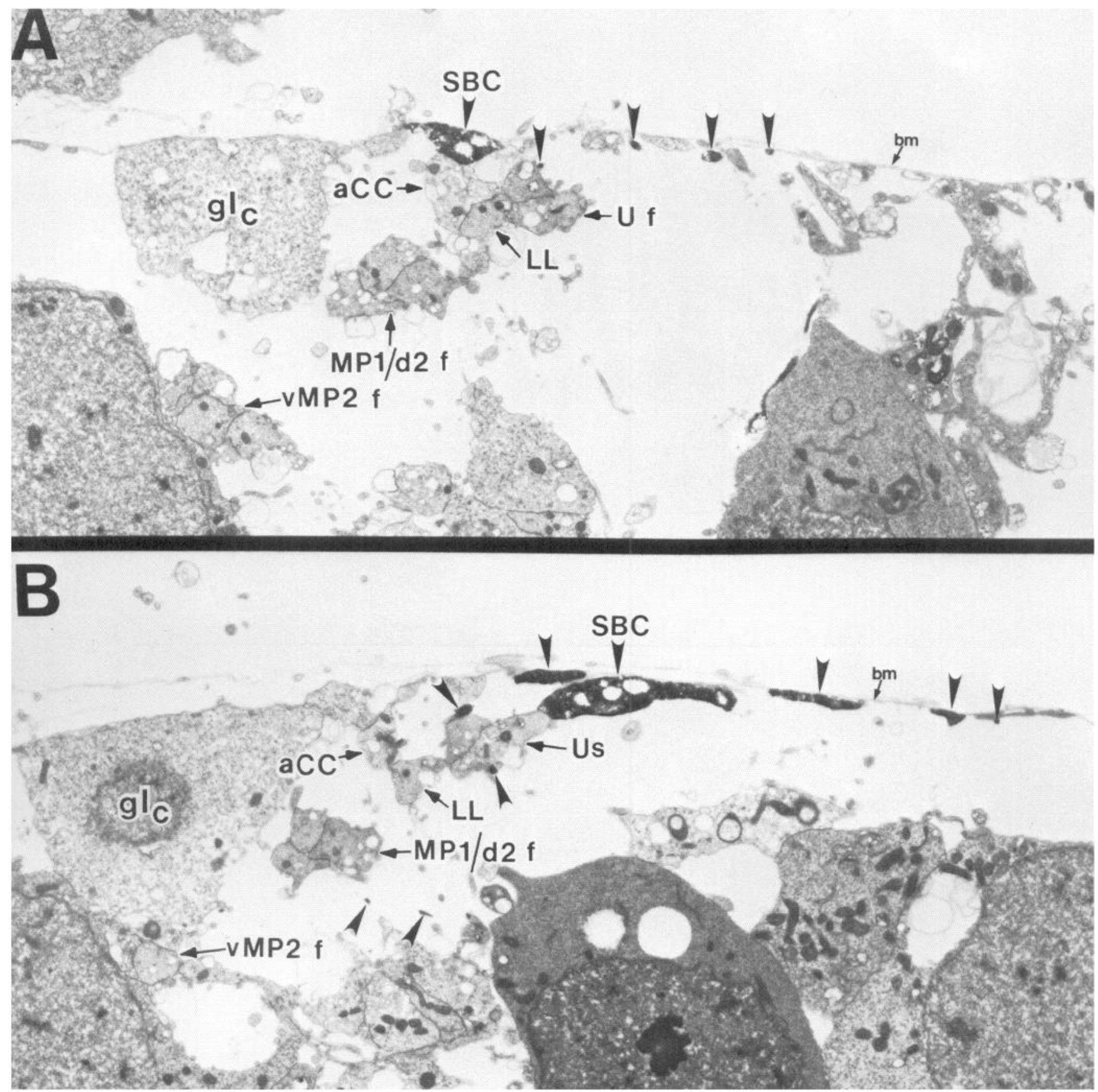

Figure 4. Glial processes along the longitudinal pathways. Four representative electron micrographs through the developing longitudinal connectives at levels A-D in Figure 3. The SBC has been filled with HRP. Section $A$ is about $20 \mu \mathrm{m}$ anterior to the cell body of the $S B C$. At this stage of development (34\%), the U growth cones $(U s)$ are just turning laterally along the SBC to pioneer the IS nerve; the $a C C$ growth cone has just turned posteriorly along the $\mathrm{U}$ axons. These 4 sections $(A-D)$ reveal the numerous processes and filopodia of the SBC and the connective glia $\left(g l_{c}\right)$ just

\section{Results}

The intersegmental nerve forms along the segment boundary cell In the first paper of this series, we described the development of the first 3 longitudinal axon fascicles connecting the developing segmental ganglia in the grasshopper embryo; these 3 fascicles initially contain the axons of 7 identified neurons. In addition to pioneering the first 3 longitudinal fascicles, 3 of these first 7 growth cones are also involved in the establishment of 1 of the 2 major peripheral nerve roots that exits the CNS, the intersegmental (IS) nerve. The axons of the $U$ fascicle turn laterally near the segment border to pioneer the IS nerve (see Fig. 1 in paper I). Thus, this peripheral nerve root is initially pioneered by the growth cones of motoneurons leaving the CNS. This nerve root is both sensory and motor; ultimately, the IS nerve contains the axons of many additional motoneurons and many peripheral sensory neurons.

The $U$ fascicle is pioneered by the $U 1$ and $U 2$ axons. The Us (U1 and U2) send their growth cones up to the dorsal basement membrane (32\% of embryonic development). They extend posteriorly about $70 \mu \mathrm{m}$ along the dorsal basement membrane (33\%). 

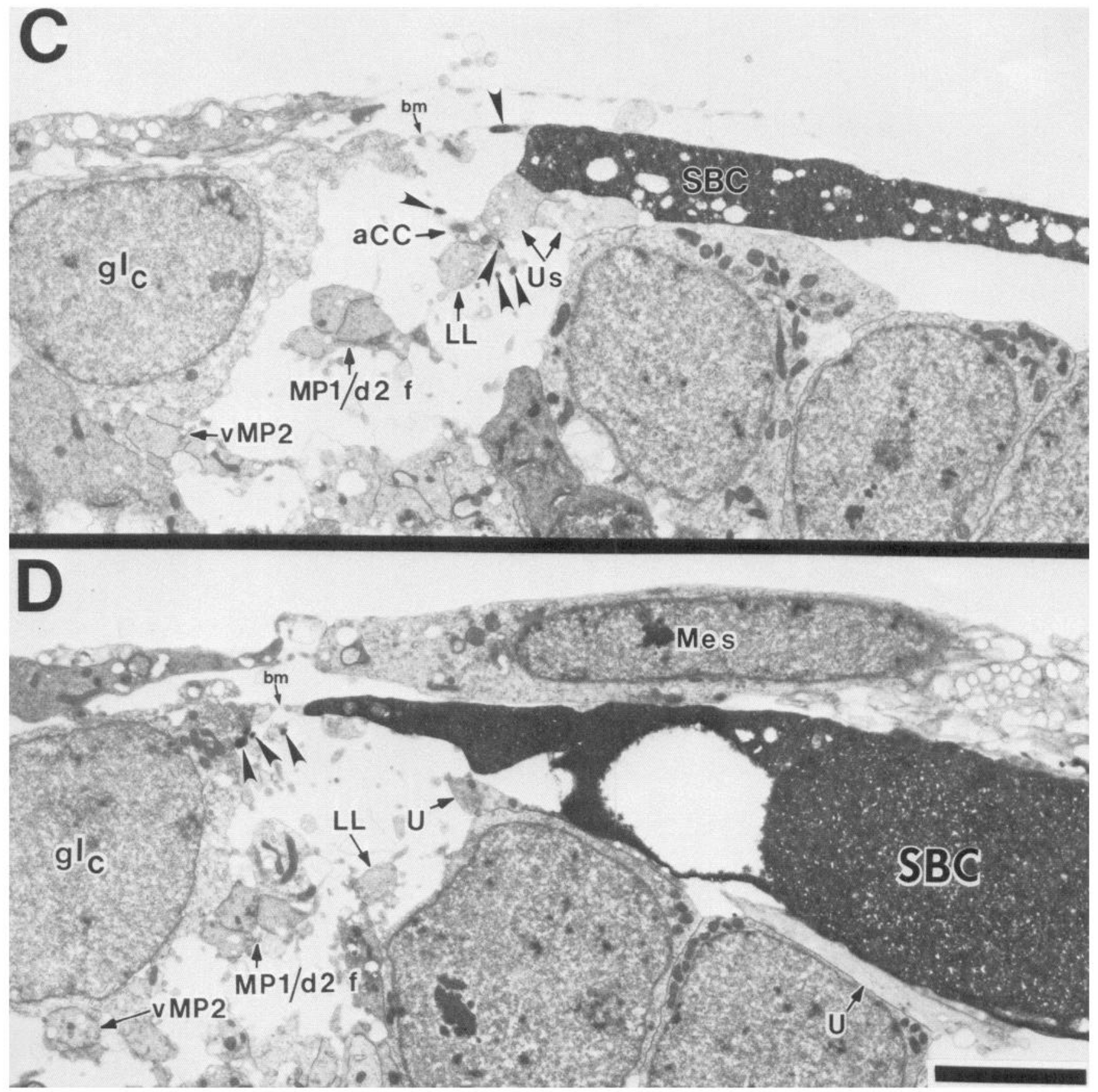

under the basement membrane $(b m)$ along the connective pathway. They also reveal the selective affinities of the first growth cones for these primitive glial cells. For example, processes of the $S B C$ extend anteriorly; the axons of the $U$ fascicle (U f) (including the aCC growth cone) are in contact with these processes along their length. For further details, see text. Scale bar, $5 \mu \mathrm{m}$.

At this stage of development, the aCC growth cone displays a selective affinity for the surface of the U axons (paper II); the $\mathrm{aCC}$ growth cone turns toward and then posteriorly along the $\mathrm{U}$ axons $(34 \%)$.

When the U growth cones reach the segment border, they turn laterally to pioneer the IS nerve (34\%). The $U$ growth cones turn laterally along the surface of a specific non-neuronal cell, the SBC, as shown in Figure $1, B-D$; as described below, the $\mathrm{SBC}$ is a primitive glial cell. Similarly, when the aCC growth cone reaches the segment border, it too turns laterally $(35 \%)$. However, although it extends posteriorly by fasciculating with the $\mathrm{U}$ axons, it sometimes leaves their surface at the segment border and displays a higher affinity for the SBC (Fig. $2 A$ ). Most growth cones, however, display no selective affinity for the SBC. For example, the MP1, dMP2, and vMP2 growth cones extend longitudinally at the segment border through the same channel as do the $\mathrm{U} 1, \mathrm{U} 2$, and aCC growth cones, without displaying any selective affinity for the SBC (Fig. $2 A$ ).

At this stage of development, the SBC is a flattened cell just under the dorsal basement membrane, with numerous processes and filopodia extending both laterally from its distal edge and longitudinally from its proximal side. The IS nerve forms di- 


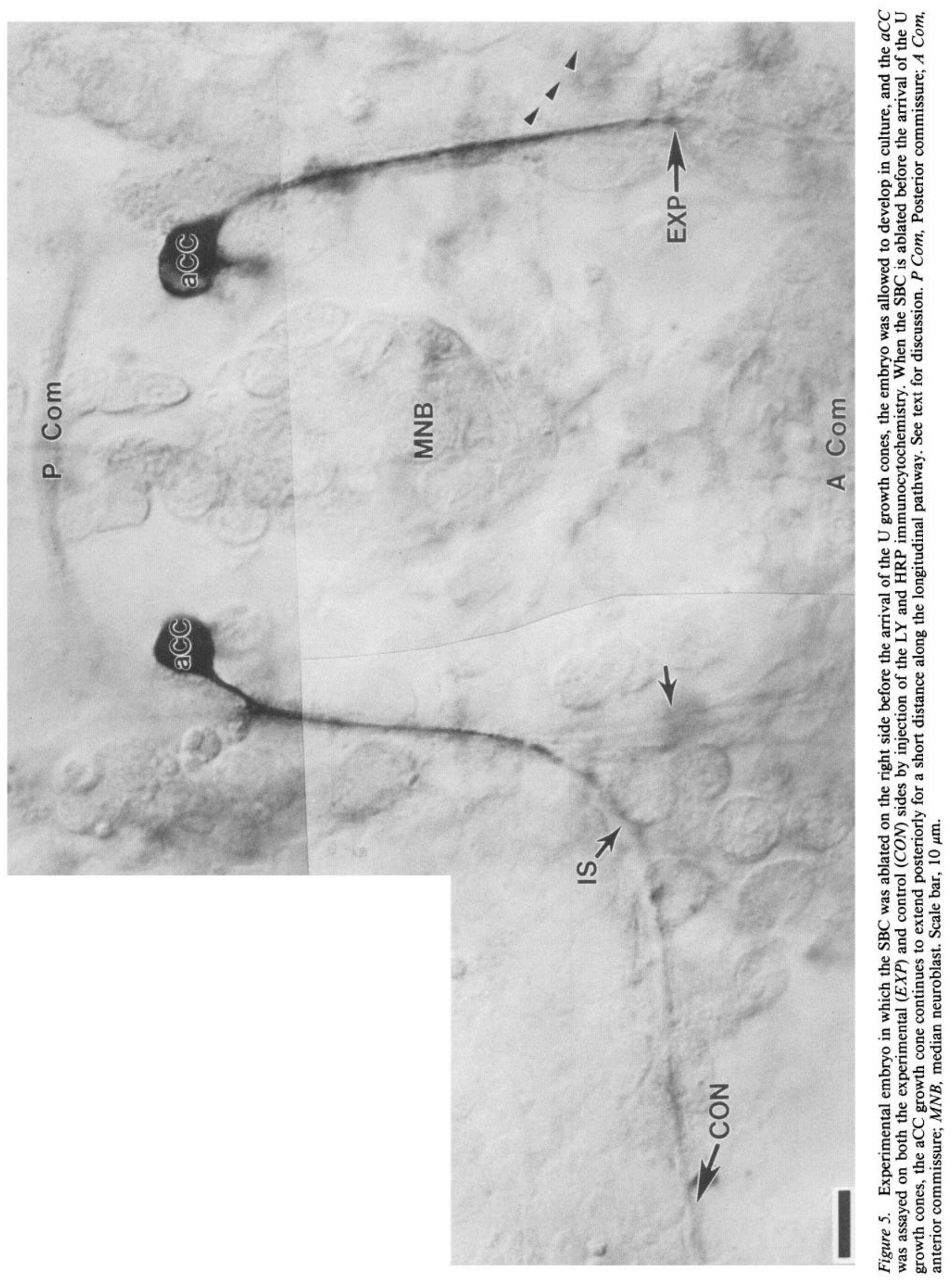


rectly along the surface of the SBC. (Figs. $1 B, 2 A$ ). As the IS nerve forms, the $\mathrm{SBC}$ changes its shape as it withdraws many of its processes and becomes more tightly aligned along the nerve (compare Fig. 1, $B$ with $D$ ).

As the $\mathrm{U} 1, \mathrm{U} 2$, and aCC growth cones continue to extend distally and exit the CNS, they leave the surface of the SBC and next extend along the surface of another identified cell (Fig. 2B). This second non-neuronal cell, called the $\mathrm{SBC}_{2}$, is also aligned along the presumptive IS nerve pathway just distal to the $\mathrm{SBC}_{1}$. Other, similar cells appear to be aligned further distally, but we have not followed the IS nerve beyond these first two.

\section{The segment boundary cell is a primitive glial cell}

The $\mathrm{SBC}$ and its distal sibling $\mathrm{SBC}_{2}$ are primitive glial cells. The best evidence for their glial phenotype is that, later in development, they differentiate into mature glial cells and enwrap the axons of the IS nerve (Fig. 2C). Also characteristic of their glial phenotype is their continued cell division. Between 35 and $40 \%$ of development, after the first growth cones have already extended along their surfaces, both of these cells have been observed in the process of dividing. After division, 1 cell remains in the original position, while the other daughter migrates distally along the nerve pathway to become a distal glial cell of the IS nerve.

We are less certain about the origin of these primitive glial cells. There are 2 possibilities. First, preliminary observations suggest that some primitive glial cells arise from a glial precursor cell which transiently appears at the lateral edge of the neuroblast (NB) plate between NB rows 2 and 3 (see Doe and Goodman, 1985). However, because these cells continue to divide and migrate, we are uncertain as to just which glial cells arise from this lineage and which arise from other precursors. Second, at the earliest ages that the SBC can be identified, it often exhibits shapes intermcdiatc between a neuroepithelial cell (Doe and Goodman, 1985) and a glial cell. At this early stage, the SBC often has a more ventrally located nucleus and a large ventral process among the neuroepithelial cell processes, in addition to its characteristic large, dorsally flattened process. This observation suggests that the $\mathrm{SBC}$, and perhaps other primitive glial cells, may arise from undifferentiated neuroepithelial cells. An accurate account of the origins of these and other glial cells in insect embryos awaits specific glial markers, such as monoclonal antibodies, that will allow their lineages and migrations to be unambiguously observed.

\section{Glial cells and processes along the longitudinal pathways}

While the SBC is aligned along the mediolateral axis near the segment border, other primitive glial cells are aligned along the anteroposterior axis along the pathway where the longitudinal connectives form; we call the latter cells glia of the connective (gl) (Fig. 1A), in contrast to the SBC and its siblings, which are glia of the intersegmental nerve $\left(\mathrm{gl}_{\mathrm{IS}}\right)$. These cells are also identified as primitive glial cells because later in development they too take on a special relationship with the axons of the longitudinal connectives (see Fig. $2 B$ in paper I).

We injected LY into the MP1 neuron and one of the $\mathrm{gl}_{\mathrm{c}}$ in embryos of different stages between 30 and $32 \%$ of development to examine their relationship to one another. Interestingly, these primitive glial cells are positioned in a preformed longitudinal pathway before the first growth cones arrive. Moreover, the first growth cones appear to interact with the processes of these glial cells in a highly specific way.

As we reported in paper I of this series, the MP1 filopodia display selective affinities for specific neuronal surfaces. However, in addition to their contact with neuronal surfaces, the MPI filopodia are also in contact with other cellular surfaces. As shown by the large arrows in Figure $6 B$ in paper I, many large processes under the basement membrane are not filopodia
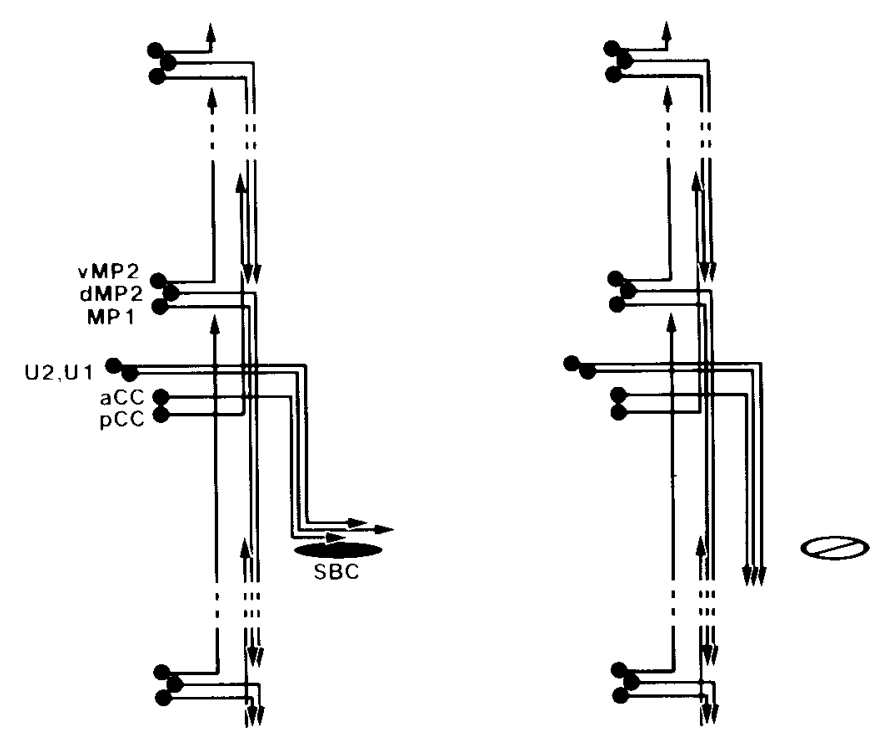

Figure 6. Schematic diagram summarizing the first 3 longitudinal axon fascicles and the IS nerve in the grasshopper embryo, and results of the in vitro ablation of the $S B C$. These results demonstrate the selective affinity of the $U 1, U 2$, and $a C C$ growth cones for the $\mathrm{SBC}$, a primitive glial cell that is part of a preformed glial pathway for the IS nerve. For further discussion, see text.

but, rather, are likely to be extensions of the primitive glial cells. Moreover, many of the filopodia that the MP1 filopodia contact are likely to originate from these glial cells as well. Although this reconstruction does not extend far enough to allow us to preciscly identify which processes and filopodia arc from the $\mathrm{gl}_{\mathrm{c}}$, it does provide suggestive evidence that the growth cones that pioneer the first longitudinal pathways might be interacting specifically with processes extending from primitive glial cells, just as the IS nerve is pioneered along a preformed glial pathway.

To better understand the relationship of glial proccsses to the axons in the first longitudinal fascicles, we filled the SBC with HRP (Fig. 3) and followed the processes of the SBC and the $\mathrm{gl}_{\mathrm{c}}$ in serial sections of the developing longitudinal connectives (Fig. 4). Four representative sections, taken at locations A-D in Figure 3, are shown in Figure 4. Section A is about $20 \mu \mathrm{m}$ anterior to the cell body of the SBC. At this stage of development (34\%), the U growth cones are just turning laterally along the SBC to pioneer the IS nerve; the aCC growth cone has just turned posteriorly along the $\mathrm{U}$ axons.

These 4 sections reveal the numerous processes and filopodia of the $\mathrm{SBC}$ and the $\mathrm{gl}_{\mathrm{c}}$ just under the basement membrane, along the connective pathway. They also reveal the selective affinities of the first growth cones for these primitive glial cells. For example, processes of the SBC extend anteriorly; the axons of the $\mathrm{U}$ fascicle are in contact with these processes along their length. The axons of the vMP2 fascicle are more ventral and are in contact with the ventral surface of the $\mathrm{gl}_{c}$; in this portion of the developing connective, the vMP2 has never been observed in contact with the dorsal basement membrane but is always seen ventrally in contact with the $\mathrm{gl}_{\mathrm{c}}$ (see also Fig. $2 A$ in paper I). The MP1 and dMP2 growth cones have extended posteriorly into the next segment by this stage of development. Nevertheless, in the most posterior of these sections (Fig. 4D) they can be seen in contact with the $\mathrm{gl}_{\mathrm{c}}$.

What is perhaps most striking is the identification in Figure $4 A$ of extensive glial processes from both the SBC and $\mathrm{gl}_{\mathrm{c}}$ just under the basement membrane along the longitudinal pathways; nearly all of the non-neuronal processes under the basement membrane belong to these glial cells. These data suggest that 
the $\mathrm{gl}_{\mathrm{c}}$ may play an important role in the establishment of the first longitudinal axonal pathways. Furthermore, they also show that the $\mathrm{U}$ growth cones, and later the aCC growth cone, contact processes of the SBC while they are still in the connective extending posteriorly. These glial processes may guide the growth cones along their lateral position (relative to the MP1/dMP2 fascicle) in the longitudinal connective, and then as they turn laterally along the SBC to pioneer the IS nerve.

\section{Cell ablation experiments}

While the data on the $\mathrm{gl}_{c}$ are only suggestive, the best evidence for a preformed glial pathway involves the formation of the IS nerve. The $\mathrm{U} 1, \mathrm{U} 2$, and aCC growth cones all display a selective affinity for the surface of the SBC, a primitive glial cell. To test whether the SBC is necessary for the formation of this axonal pathway, we used a microelectrode to ablate the SBC before the arrival of the $U$ growth cones and then allowed the embryos to develop in embryo culture (for ablation and culturing methods, see paper I).

When the SBC is ablated before the arrival of the $U$ growth cones $(n=7)$, the aCC growth cone either stops growing at the level of the IS nerve or continues to extend posteriorly for a short distance along the longitudinal pathway. It does not turn laterally in the absence of the SBC. An example of such an experiment is shown in Figure 5.

In embryos older than $40 \%$, in which the SBC was ablated at $33-34 \%$, a much diminished IS nerve can be seen. One possibility is that this diminished IS nerve may represent only the ingrowing sensory neurons, which may not require the SBC to reach the CNS. Alternatively, the SBC may have been replaced, thus providing guidance for later-developing motoneurons.

\section{Discussion}

During the development of the vertebrate nervous system, pioneering growth cones have a special relationship with primitive glial cells in a variety of locations, including the developing spinal cord (e.g., Kuwada, 1986; Singer et al., 1979) and the developing nerve roots that exit the spinal cord (Nordlander et al., 1981). In this paper, we have shown a similar relationship between pioneering growth cones and primitive glial cells in the developing longitudinal connectives and peripheral nerve roots of the grasshopper embryo.

We have shown the role of a preformed glial pathway in the formation of the IS nerve, one of the major roots for both motoneurons and sensory neurons exiting and entering the CNS. The U1 and $\mathrm{U} 2$ growth cones pioneer the IS nerve. They and the aCC growth cone display a selective affinity for the SBC, a primitive glial cell that establishes the location of this nerve root. Similar primitive glial cells, called connective glia, are also found along the pathway where the longitudinal connectives form, and they too may play an important role in the formation of the first longitudinal axonal pathways. Experimental analysis shows that, when the SBC is ablated, the growth cones that normally turn laterally to pioneer the IS nerve do not do so; this confirms the important guidance role played by this glial cell (Fig. 6).

Similar primitive glial cells exist in the Drosophila embryo as well, and may play a similar role in growth-cone guidance. We previously showed that, in general, the growth cones of homologous neurons display the same selective affinities for specific axonal surfaces in the Drosophila embryo as in the grasshopper embryo (Bastiani et al., 1985a; Goodman et al., 1984; Thomas et al., 1984). The similarities between these 2 insects extend to the primitive glial cells as well. In Drosophila, the U fascicle turns laterally along the homolog of the SBC.

There is, however, one interesting difference (Bastiani et al., 1985a; Goodman et al., 1984). In the grasshopper embryo, the aCC cell body is about $70 \mu \mathrm{m}$ anterior to the SBC; it initially follows the $\mathrm{U}$ axons posterior, and then turns laterally along the SBC. In the Drosophila embryo, the aCC cell body is about 7 $\mu \mathrm{m}$ anterior to the SBC. Just as in the grasshopper, in the fly the SBC has processes extending anteriorly along the longitudinal pathway. Also as in the grasshopper, in the fly the aCC extends laterally along the SBC. However, in the fly embryo, the aCC growth cone, instead of waiting for the $U$ axons, immediately extends toward and along the SBC, pioneering the IS ncrve. The aCC appcars to be in direct contact with processes of the SBC from the outset, since a process of the SBC extends anteriorly and contacts the aCC cell body. Furthermore, the aCC axon appears to run posteriorly along the SBC process. Once the $\mathrm{U}$ growth cones arrive, they fasciculate with the $\mathrm{aCC}$ axon.

We postulate that a simple, orthogonal scaffold of primitive glial cells is involved in the initial patterning of axonal pathways within and exiting the insect CNS. As development proceeds, the complexity of axonal pathways and fascicles in the CNS increases dramatically. It appears that 2 types of interactions are involved in the unfolding of this orthogonal scaffold of axon fascicles. In the first, neuronal growth cones interact with primitive glia to form the first axonal pathways. In the second, neuronal growth cones interact much more specifically with other neurons, displaying selective affinities for specific axonal surfaces (e.g., papers I and II). In this way a complex pattern of axon fascicles unfolds by a series of specific interactions, first between neurons and glia and then between neurons and other neurons.

At the location where the IS nerve forms, the pioneering growth cones contact 2 different glial pathways, the SBC and the $\mathrm{gl}_{\mathrm{c}}$, and yet make cell-specific and often divergent choices of which way to go. The MP1, dMP2, and vMP2 growth cones establish 2 different longitudinal axon pathways, while the U1, $\mathrm{U} 2$, and aCC growth cones turn laterally along the SBC to pioneer the IS nerve. These choices suggest some degree of specificity, allowing growth cones to distinguish between different glial pathways. However, the degree of molecular heterogeneity required for the recognition of glial pathways is considerably less than that required for the highly selective recognition of specific axonal surfaces.

In vertebrates, cell-adhesion molecules such as N-CAM (Edelman, 1983; Noble et al., 1985; Rutishauser, 1984; Silver and Rutishauser, 1984) and J1 (Kruse et al., 1985) are expressed on the surfaces of glial cells and appear to play a major role in neuron-glial adhesion. These types of adhesion molecules would be good candidates as the molecules involved in growth-cone guidance along the surfaces of the primitive glial cells in insects. Using the cellular and molecular genetic attributes of grasshopper and Drosophila embryos, respectively, it should be possible in the future to elucidate the molecular mechanisms involved in the general recognition of glial pathways early in neuronal development, and in the more specific recognition of axonal pathways that occurs shortly thereafter.

\section{References}

Bastiani, M. J., and C. S. Goodman (1984a) Neuronal growth cones: Specific interactions mediated by filopodial insertion and induction of coated vesicles. Proc. Natl. Acad. Sci. USA 81: 1849-1853.

Bastiani, M. J., and C. S. Goodman (1984b) The first growth cones in the central nervous system of the grasshopper embryo. In Cellular and Molecular Approaches to Neuronal Development, I. Black, ed., pp. 63-84, Plenum, New York.

Bastiani, M. J., J. A. Raper, and C. S. Goodman (1984) Pathfinding by neuronal growth cones in grasshopper embryos. III. Selective affinity of the $G$ growth cone for the $P$ cells within the A/P fascicle. J. Neurosci. 4: 2311-2328.

Bastiani, M. J., C. Q. Doe, S. L. Helfand, and C. S. Goodman (1985a) 
Neuronal specificity and growth cone guidance in grasshopper and Drosophila embryos. Trends Neurosci. 8: 257-266.

Bastiani, M. J., S. du Lac, and C. S. Goodman (1985b) The first neuronal growth cones in insect embryos: Model system for studying the development of neuronal specificity. In Model Neural Networks and Behavior, A. I. Selverston, ed., pp. 149-174, Plenum, New York.

Bastiani, M. J., S. du Lac, and C. S. Goodman (1986) Guidance of neuronal growth cones in the grasshopper embryo. I. Recognition of a specific axonal pathway by the pCC neuron. J. Neurosci. $6: 3518-$ 3531.

Bate, C. M. (1976) Embryogenesis of an insect nervous system. I. A map of the thoracic and abdominal neuroblasts in Locusta migratoria. J. Embryol. Exp. Morphol. 35: 107-123.

Bate, C. M., and E. B. Grunewald (1981) Embryogenesis of an insect nervous system. II. A second class of precursor cells and the origin of the intersegmental connectives. J. Embryol. Exp. Morphol. 61: $317-330$

Bentley, D., and M. Caudy (1983) Navigational substrates for peripheral pioneer growth cones: Limb-axis polarity cues, limb-segment boundaries, and guidepost neurons. Cold Spring Harbor Symp. Quant. Biol. 48: 573-585.

Bentley, D., and H. Keshishian (1982) Pathfinding by peripheral pioneer neurons in grasshoppers. Science 218: 1081-1088.

Berlot, J., and C. S. Goodman (1984) Guidance of peripheral pioneer neurons in the grasshopper: An adhesive hierarchy of epithelial and neuronal surfaces. Science 223: 293-295

Blair, S. S., and J. Palka (1985a) Axon guidance in cultured wing discs and disc fragments of Drosophila. Dev. Biol. 108: 411-419.

Blair, S. S., and J. Palka (1985b) Axon guidance in the wing of Drosophila. Trends Neurosci. 8: 284-288.

Carney, P. R., and Silver, J. (1983) Studies on cell migration and axon guidance in the developing distal auditory system of the mouse. J. Comp. Neurol. 215: 359-369.

Doe, C. Q., and C. S. Goodman (1985) Early events in insect neurogenesis: I. Development and segmental differences in the pattern of neuronal precursor cells. Dev. Biol. 111: 193-205.

du Lac, S., M. J. Bastiani, and C. S. Goodman (1986) Guidance of neuronal growth cones in the grasshopper embryo. II. Recognition of a specific axonal pathway by the aCC neuron. J. Neurosci. 6: 35323541 .

Edelman, G. M. (1983) Cell adhesion molecules. Science 219: 450457.

Goodman, C. S., J. A. Raper, R. K. Ho, and S. Chang (1982) Pathfinding of neuronal growth cones in grasshopper embryos. In Developmental Order: Its Origin and Regulation, S. Subtelny and P. B. Green, eds., pp. 275-316, Liss, New York.

Goodman, C. S., M. J. Bastiani, C. Q. Doe, S. du Lac, S. L. Helfand, J. Y. Kuwada, and J. B. Thomas (1984) Cell recognition during neuronal development. Science 225: 1271-1279.

Ho, R. K., and C. S. Goodman (1982) Peripheral pathways are pioneered by an array of central and peripheral neurones in grasshopper embryos. Nature 297: 404-406.

Jan, Y. N., A. Ghysen, I. Christoph, S. Barbel, and L. Y. Jan (1985) Formation of neuronal pathways in the imaginal discs of Drosophila melanogaster. J. Neurosci. 5: 2453-2464.
Kruse, J., G. Keilhauer, A. Faissner, R. Timpl, and M. Schachner (1985) The Jl glycoprotein: A novel nervous system adhesion molecule of the L2/HNK-1 family. Nature 316: 146-148.

Kuwada, J. Y. (1986) Cell recognition by neuronal growth cones in a simple vertebrate embryo. Science 233: 740-746.

Nardi, J. B. (1983) Neuronal pathfinding in developing wings of the moth Manduca sexta. Dev. Biol. 95: 163-174.

Noble, M., M. Albrechtsen, C. Moller, J. Lyles, E. Bock, C. Goridis, M. Watanabe, and U. Rutishauser (1985) Glial cells express N-CAM/ D2-CAM-like polypeptides in vitro. Nature 316: 725-728.

Nordlander, R. H., J. F. Singer, R. Beck, and M. Singer (1981) An ultrastructural examination of early ventral root formation in amphibia. J. Comp. Neurol. 199: 535-551.

Raper, J. A., M. J. Bastiani, and C. S. Goodman (1983a) Pathfinding by neuronal growth cones in grasshopper embryos. I. Divergent choices made by the growth cones of sibling neurons. J. Neurosci. 3: 20-30.

Raper, J. A., M. J. Bastiani, and C. S. Goodman (1983b) Pathfinding by neuronal growth cones in grasshopper embryos. II. Selective fasciculation onto specific axonal pathways. J. Neurosci. 3: 31-41.

Raper, J. A., M. J. Bastiani, and C. S. Goodman (1983c) Guidance of neuronal growth cones: Selective fasciculation in the grasshopper embryo. Cold Spring Harbor Symp. Quant. Biol. 48: 587-598.

Raper, J. A., M. J. Bastiani, and C. S. Goodman (1984) Pathfinding by neuronal growth cones in grasshopper embryos. IV. The effects of ablating the $A$ and $P$ axons upon the behavior of the $G$ growth cone. J. Neurosci. 4: 2329-2345

Rutishauser, U. (1984) Developmental biology of a neural cell adhesion molecule. Nature 310: 549-554.

Silver, J., and M. Y. Ogawa (1983) Postnatally induced formation of the corpus callosum in acallosal mice on glia-coated cellulose bridges. Science 220: 1067-1069.

Silver, J., and U. Rutishauser (1984) Guidance of optic axons in vivo by a preformed adhesive pathway on neuroepithelial endfeet. Dev. Biol. 106: 485-499.

Silver, J., and J. Sapiro (1981) Axonal guidance during development of the optic nerve: The role of pigmented epithelia and other extrinsic factors. J. Comp. Neurol. 202: 521-538.

Silver, J., and R. L. Sidman (1980) A mechanism for the guidance and topographic patterning of retinal ganglion cell axons. J. Comp. Neurol. 189: 101-111.

Silver, J., S. E. Lorenz, D. Wahlsten, and J. Coughlin (1982) Axonal guidance during development of the great cerebral commissures: Descriptive and experimental studies, in vivo, on the role of preformed glial pathways. J. Comp. Neurol. 210: 10-29.

Singer, M., R. H. Nordlander, and M. Egar (1979) Axonal guidance during embryogenesis and regeneration in the spinal cord of the newt. The blueprint hypothesis of neuronal pathway patterning. J. Comp. Neurol. 185: 1-22.

Taghert, P., M. J. Bastiani, R. K. Ho, and C. S. Goodman (1982) Guidance of pioneer growth cones: Filopodial contacts and coupling revealed with an antibody to Lucifer Yellow. Dev. Biol. 94: 391-399.

Thomas, J. B., M. J. Bastiani, C. M. Bate, and C. S. Goodman (1984) From grasshopper to Drosophila: A common plan for neuronal development. Nature 310: 203-207. 